NASA-TM-111579

\title{
Plate mode velocities in graphite/epoxy plates
}

\author{
W. H. Prosser

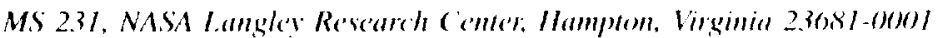

\author{
M. R. Gorman

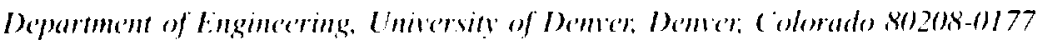

(Received 20 July 1993; revised 29 March 1994; accepted 2 May 1994)

\begin{abstract}
Measurements of the velocities of the extensional and flexural plate modes were made along three directions of propagation in four graphite/epoxy composite plates. The acoustic signals were generated by simulated acoustic emission events (pencil lead breaks or Hsu-Neilsen sources) and detected by broadband ultrasonic transducers. The first arrival of the extensional plate mode, which is nondispersive at low frequencies. was measured at a number of different distances from the source along the propagation direction of interest. The velocity was determined hy plolting the distance versus arrival time and computing its slope. Because of the large dispersion of the flexural mode, a Fourier phase velocity lechnigue was used to characterize this mode. The velocity was measured up 10 a frequency of $160 \mathrm{kHz}$. Theoretical predictions of the velocilies of these modes were also made and compared with experimental observations. Classical plate theory yielded good agreement with the measured extensional velocities. For predictions of the dispersion of the flexural mode, Mindlin plate theory. which includes the effects of shear deformation and rotatery inertia was shown to give better agreement with the experimental measurements.
\end{abstract}

PACS numbers: 43.40 .1$) \times$

\section{INTRODUCTION}

Acoustic signals propagate in thin plates as the extensiomal and flexural plate modes when the wavelength of the acoustic signal is much larger than the plate thickness. This fact is of importance in a number of nondestructive evaluattion (NDE) techniques that use acoustic waves such as the acoustic emission (AE) technique. The propagation of $\mathrm{AE}$ signals as plate modes has heen demonstrated by a number of researchers. It was shown using simulated $\mathrm{AE}$ sources (pencil lead breaks) by Gorman' on thin aluminum and gr/ep composite plates and hy (iorman and Prosser ${ }^{2}$ on thin aluminum plates. A typical signal from a simulated AE source (pencil lead break) is shown in Fig. 1. The extensional and flexural mode conmonents of this waveform are identified in this figure. AE signals from transverse matrix cracking sources in gr/ep composite plates were also shown to propagate as plate modes by Gorman and Ziolat. ${ }^{3}$ Smith $^{4}$ showed that crack growth events in thin aluminum plates under spectrum fatigue loading produced signals that propagated as plate modes.

The propagation of acoustic signals as plate modes in thin plates also occurs in other NDE techniques such as conventional ultrasonic testing. acousto-ultrasonics, and laser generaled ultrasonics. Duke of al. ${ }^{5}$ suggested that the acousto-ultrasonic technique generated plate modes. made tone burst measurements of the velocities of the plate modes. and compared them with predictions based on classical plate theory (CPT). Schumacher et al." demonstrated the existence of plate modes in laser generated ultrasonic signals in thin plates which were detected by noncontacting laser interferometers. They also made measurements of the dispersion of these two modes in steel plates and compared them with theory and finite element modeling.
For all of these NDE techniques, the propagation characteristics of these plate modes ate of great importance. In acoustic emission testing. Gorman' discussed how crroneous source focation could be obtained using conventional first threshold crossing or peak arrival lechniques because of the presence of plate modes which propagate with different and dispersive velocilies. Such source location errors were substantiated by Ziola and Gorman and an allernative method for source location based on cross-correlation of the flexural mode waves was demonstrated. In the other mentioned NDE teclmiques, measurements of the velocities of the plate modes are useful for determining material properties and locating haws. Mal ot al., Veidt and Sayir, and Dean" have discussed how measurements of flexural mode dispersion might he useful in determining the elastic constants of composite plates.

In this research, measurements were made of the extensional and flexural plate mode velocities on four different composite laminates in three directions of propagation. The ply layups for these laminates were $\left[0_{10}\right],\left[0_{4}, 90_{4}\right]$. $[0.90]_{4}$, and $[0.45,-45.90]_{2,}$. Pencil lead breaks (HsuNeilsen sources) were used to generate the acoustic signals which were detected by wide hand ultrasonic sensors. The first arrival time of the extensional mode as a function of distance from the source was used to compute its velocity. A Fourier phase technique was used to determine the flexural mode dispersion up to a frequency of $160 \mathrm{kHz}$. The measurements were made along the two principal axes $(0$ and 90$)$ deg) in the plane of the plate and at an angle of $45 \mathrm{deg}$.

The ability of existing plate theories for predicting the measured plate-mode velocities was also investigated. CPT wass successfully used to predict the velocity of the extensional plate mode using clastic moduli calculated from laminated plate theory. The experimentally measured flexural 


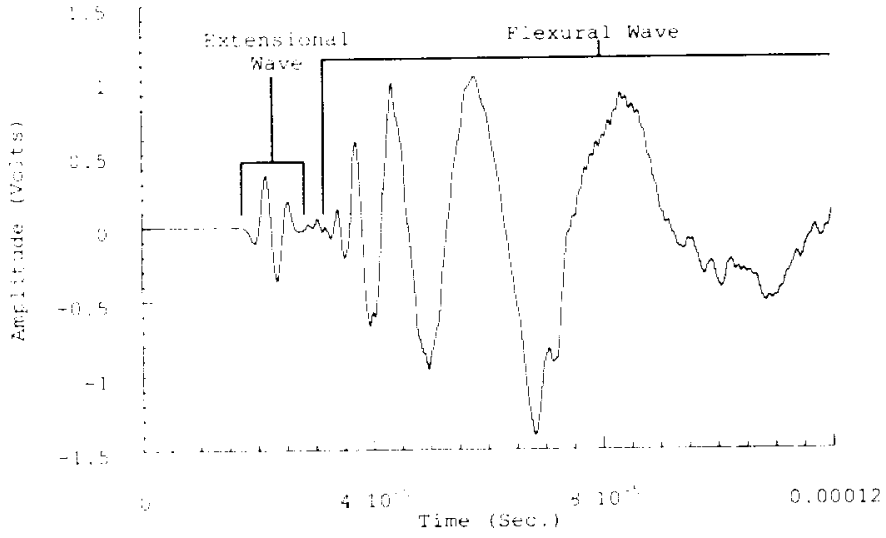

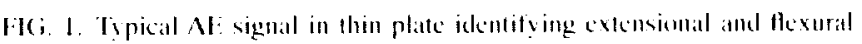
plate modes.

mode dispersion curves were also compared with theoretical predictions based on ( $P$ T. The lack of agreement between theory and experiment at the higher frequencies demonstrated the limitations of ('PT for composite materials. These are calused by the effects of shear deformation and rotatory inertia which are neglected in (P'T. Mindlin plate theory which includes these effects wats then used to predict the dispersion behavior for this mode. These predictions were in much better agreement with the experimental flexural mode dispersion measurements.

\section{THEORY}

In this research, two theoretical approaches were used for predicting the hehavior of plate mode waves in gr/ep composite laminates. The first theoretical predictions were hased on ( PT. This is a widely used approximalc theory for describing motion in thin plates where the wavelength $(\lambda)$ is much larger than the plate thickness $(h)$. A number of aluthors have presented (PT in detail including Graff ${ }^{11}$ who derives the equations of motion for isotropic materials and Whitne ${ }^{12}$ who includes the effects of anisotropy. For extensional mode waves in a symmetric orthotropic laminate, the predictions of the velocity are derived from the equations of motion for in-plane displacements which are given by

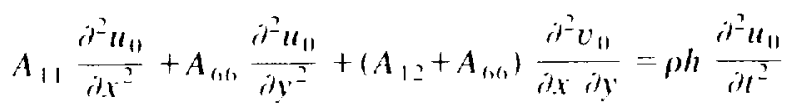

and

$$
\begin{aligned}
& A_{22} \frac{\partial^{2} v_{11}}{\partial^{2}}+A_{n o} \frac{\partial^{2} v_{11}}{\partial x^{2}}+\left(A_{12}+A_{0 n}\right) \frac{\partial^{2} u_{11}}{\partial x \partial y} \\
& =\rho h \frac{\partial^{2} v_{n}}{\partial t^{2}} .
\end{aligned}
$$

In the previous equations, $u_{11}$ and $v_{11}$ are the midplane displacements along the $x$ and $y$ axes which are orthogonal axes in the plane of the plate, $\rho$ is the density, and the $A_{i}, 3$ are the anisotropic in-plane stiffness coefficients obtained from laminated plate theory as described by Whitney ${ }^{2}$ or Tsai and Hahn. ${ }^{13}$
These equations predict two modes of propagation. In general, one mode is quasi-extensional with the largest component of its particle displacement along the propagation direction and the oller is quasi-in-plane shear with the largest component of particle displacement perpendicular to the direction of propagation in the platre of the plate. Along symmetry directions, these modes are pure mode extensional and shear-horizontal (SH) plate modes. The dispersion relations for these two waves are determined by substituting a plane wave displacement into the equations of motion and solving the resulting christoftels equation. For propagation along the $x$ axis or 1 deg direction, this procedure produces an extensional mode that is pure mode and whose velocily $\left(c_{,}\right)$ is given by

$$
c_{1}=\sqrt{A_{11} / p h}
$$

Along the $y$ axis, the extensional mode is also pure mode and its velocity is given by

$$
c_{1}=\sqrt{A_{2} / \mu h} \text {. }
$$

For propagation al 45 deg between $x$ and $y$. the wave is quasi-extensional with a velocily given by

$$
\therefore-\sqrt{\frac{\left(A_{11}+2 A_{6,1}+A_{22}\right)+\sqrt{R}}{4 \mu h}} .
$$

where

$$
\begin{aligned}
& R-\left(A_{11}+2 A_{(10}+A_{22}\right)^{2}-4\left(A_{11}+A_{(n, 1}\right)\left(A_{22}+A_{(x, 1)}\right. \\
& +4\left(A_{12}+A_{(1,)^{2}}\right)^{2}
\end{aligned}
$$

For tlexural waves, (PT wats again used to predict the dispersion. In this casce, the plate is assumed to be under a state of pure bending in which plane sections of the plate remain plane and perpendicular to the midplane of the plate. Thus, shear deformation is not included in this theory. $\triangle$ state of plane stress is assumed and the effects of rotatory inertia are also neglected. The ('P' equation of motion for an orthotropic composite laminate in the absence of hody forces is

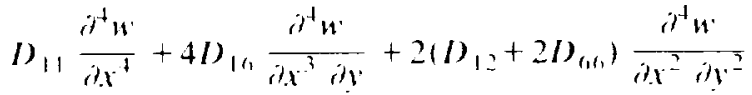

$$
\begin{aligned}
& +4 \partial_{2 k} \frac{\partial^{4} w}{\partial \lambda^{3}}+D_{22} \frac{\partial^{4} w}{\partial y^{4}}+\beta h \frac{\partial^{2} w}{\partial^{2}}=0 \text {. }
\end{aligned}
$$

where the $D_{11}$ 's are the anisotropic bending stiffness coefficients obtained from laminated plate theory as described by Whitney ${ }^{12}$ or Tsai and Hahn. ${ }^{13}$ In the previous equation, $w$ is the displacement along the $z$ axis which is normal to the plane of the plate.

The dispersion behavior for the flexural mode using CPT is also obtained by substituting the displacement for a plane wave propagating along the direction of interest into the equation of motion. After substitution and reduction of terms, the resulting (PT dispersion relation along the $x$ axis is

$$
c_{1}=\sqrt[4]{D_{11} / \rho h} \sqrt{\omega}
$$

where $c$, is the velocity of the flexural mode and $\omega$ is the angular frequency. Along the $y$ axis, the dispersion relation is 


$$
c_{1}=\sqrt[4]{D_{22} / \rho / h} \sqrt{\omega}
$$

and at 4.5 deg the dispersion relation is

$$
\begin{aligned}
c_{1}= & \sqrt[4]{\frac{\left.\left.\left.\left.\left.\frac{l}{1}(l)_{11}+4 l\right)_{11}+2(l)_{12}+2 l\right)_{11}\right)+4 l\right)_{26}+l_{22}\right)}{\rho / h}} \\
& \times \sqrt{\omega} .
\end{aligned}
$$

Unlike the predictions for the extensional mode, the flexural mode is predicted to have a velocity that is dispersive. The velocity is predicted to increase as the square root of the frequency.

Although CPT yiclded good results for the case of the extensional mode, it was found to be of limited value for the flexural mode hecause of the effects of shear deformation and rotatory inertia. Thus, a second theory was also used to predict the dispersion of the flexural mode. This theory includes the effects of shear deformation and rotatory inertia. It was put forth by Tang ef al. ${ }^{14}$ following earlier work hy Yang 't al. ${ }^{15}$ which was an extension of Mindlin plate theory. "A detailed presentation of this theory is beyond the seope of this paper. However, the dispersion behavior for a symmetric orthotropic laminate is obtatined when the determinant of the following matrix of coefficients is sel equal to zero

$$
\left[\begin{array}{lll}
M_{11} & M_{12} & M_{13} \\
M_{21} & M_{22} & M_{23} \\
M_{31} & M_{32} & M_{33}
\end{array}\right]
$$

where

$$
\begin{aligned}
& M_{11}=D_{1,} k^{2} I_{1}^{2}+2 D_{11} k^{2} I_{1} I_{1}+D_{616} k^{2} I_{1}^{2}+A_{55}-I \omega^{2} \text {. } \\
& \left.\left.M_{12}=D\right)_{10} k^{2}+\left(D_{12}+D\right)_{66}\right) k^{2} I_{1} I_{1} . \\
& M_{1,3}=i A_{5,5} k l_{1} \text {. } \\
& \left.M_{21}=D_{11} k^{2}+(I)_{12}+I_{6,1}\right) k^{2} I_{1} I_{1} \text {, } \\
& M_{22}=I_{60} k^{2} I_{1}^{2}+2 I_{16} k^{2} I_{1} I_{1}+D_{22} k^{2} I_{1}^{2}+A_{44}-I_{0}{ }^{2} \text {. } \\
& M_{2,1}=i A_{4+} k I_{1}, \\
& M_{31}=-i A_{55} k l \text {, } \\
& M_{32}=-i A_{4+} k l_{1} \text {, }
\end{aligned}
$$

and

$$
M_{33}=A_{55} k^{2} I_{1}^{2}+A_{44} k^{2} I_{1}^{2}-\rho^{*} \omega^{2} .
$$

In the previous equations, $l_{,}$and $l_{b}$ are the direction cosines for the propagation direction of interest, $k$ is the wave number,

$$
\left(\rho^{*}, h\right)=\int_{z=2}^{z-h / 2} \rho\left(1, z^{2}\right) d z
$$

and

$$
A_{i j}=\kappa_{i} \kappa_{j} \int_{z-h_{h ! 2}}^{h: 2}\left(Q_{i j}\right)_{d} d z, \quad \text { for } i, j=4.5
$$

WBBI I I I imina propertics of ASt 3502 graphite eposy.

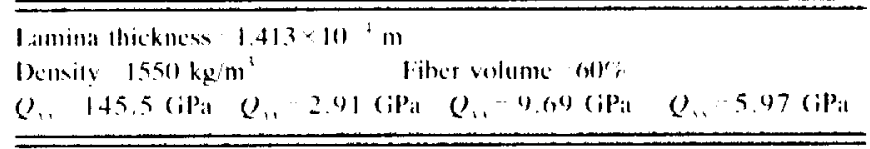

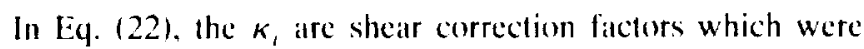
determined to yield the best agreement with threedimensional elasticity theory when $\kappa_{i}^{2}=5 / 6$. The subseript $/$ refers to the /th layer of the laminate and the $Q_{1}$, are the stiffnesses for the th layer. Solving the determinant for the wave number as a function of $\omega$ yields a cubic in $k^{2}$. Only the root which approaches zero as the frequency approaches zero is the correct root. Once $k$ as a function of $\omega$ is known. the phase velocity is determined as a function of frequency using the relation

$$
c,=\omega / k \text {. }
$$

\section{EXPERIMENT}

The composite laminates used in this study were mate of AS4/35012 graphite/epoxy. All four laminales consisted of sixteen plys and had a nominal thickness of $2.26 \mathrm{~mm}$. The dimensions were $0.50 \mathrm{~m}$ along the $x$ direction $(0)$ deg ply direction) and $0.381 \mathrm{~m}$ along the $y$ direction. Measurements were made along the 1$)$ deg ( $x$ direction), 45, and 9() deg directions for all four laminates. The nominal lamina properties for this material as obtained from the manuficturer are given in Table 1 . These values were used in the laminated plate theory calculations to obtain the in-plane and bending stiffness coefficients needed for the theoretical dispersion calculations.

For measurements of the extensional and flexural velocilics, a pencil lead bread was used to excite the acoustic walves. The waves were delected with Panametrics 3.5-Mllz broadhand ultrasonic transducers. These sensors have been shown previously by Prosser ${ }^{17}$ to provide tlat frequency, displacement sensitivity response to these low-frequency plate waves. The detected signals were preamplified by 4() $\mathrm{dB}$ with model 1220 A preamplifiers from Physical Acoustic Corporation (PAC). There were no filters used in the preamps. The amplitied signals were digitized at a sampling frequency of 5 $\mathrm{MHz}$ for the extensional measurements and $1 \mathrm{MHz}$ for the flexural measurements with a LeCroy 6810 transient recorder, and then stored on a personal computer for analysis. A high sensitivity resonant AE transducer (PAC model RI5) was positioned next to the lead break and was used to trigger the transient recorder.

For the extensional measurements, a single receiving sensor was used to detect the waves along the propagation direction of interest. The simulated AE signal was repeated five times while the receiving transducer was moved over a range of distances from the source which varied from 7.62 to $17.78 \mathrm{~cm}$ in $2.54-\mathrm{cm}$ increments. The first arrival time of the extensional mode was then determined for each of the signals from the digitized time records and plotted as a function of distance from the source. A linear least-squares tit was used to determine the slope of the curve which is the velocity of the extensional mode. This process was repeated for each 


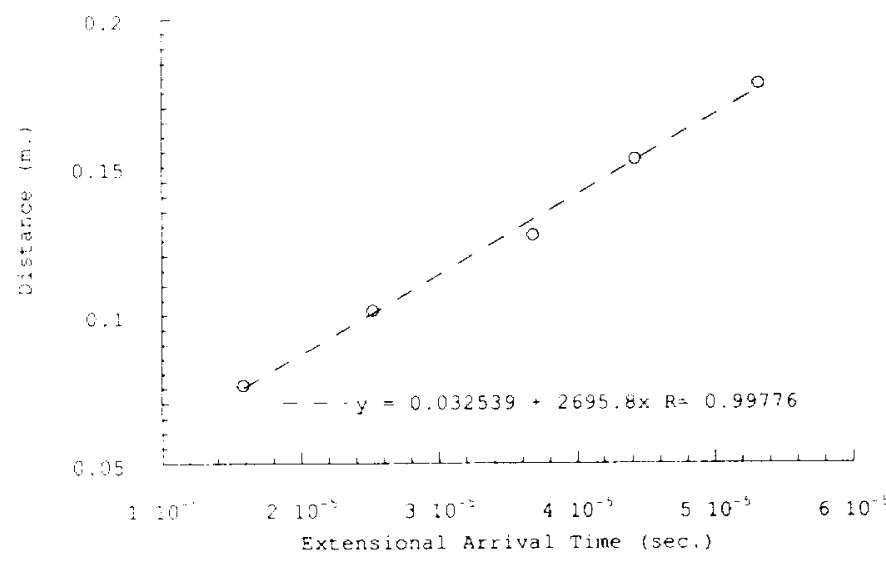

IIC. 2. Phe of extensional arrival time versels distance of propagation for g()-dege propatgation in $\left|11_{14}\right|$ graphite eposy plats.

of the three directions in the four plates. A typical plot of the extensional arrival time versus distance of propagation is shown in Fig. 2 .

A Fourier phase technique wats used for the measurement of the flexural mode dispersion. This technique has been described by a number of authors including Sachse and Pao, "' Pao and Sachse. ${ }^{19}$ Veidt and Sayir," Dean. "1" and Alleyne and (awley." In this technique, the elastic wave is detected at two different distances away from the source of the wave along the direction of propagation of interest. The phase $(\varphi)$ of the wave at each position at a given frequency $(f)$ is determined by performing a Fourier transform on the signals. The phase must be unwrapped to remove the $2 n \pi$ uncertainty. The phase difference $\left(\Delta_{\varphi}\right)$ over the distance between the two transducers ( $J_{x}$ ) is then computed for each frequency. The wave number and velocity are then calculated at each frequency by

$$
k(f)=\frac{\Delta \varphi(f)}{\Delta x}
$$

and

$$
c(f)=\frac{2 \pi f}{k(f)}=\frac{2 \pi f \Delta x}{\Delta \varphi(f)} .
$$

The experimental setup used for these experiments is shown in Fig. 3. Transducer separations of 1.91, 2.54, 3.18. 3.81 , and $4.45 \mathrm{~cm}$ were used. An average velocity and standard deviation for the five different measurements was computed. The source and receivers were kept as nearly in the

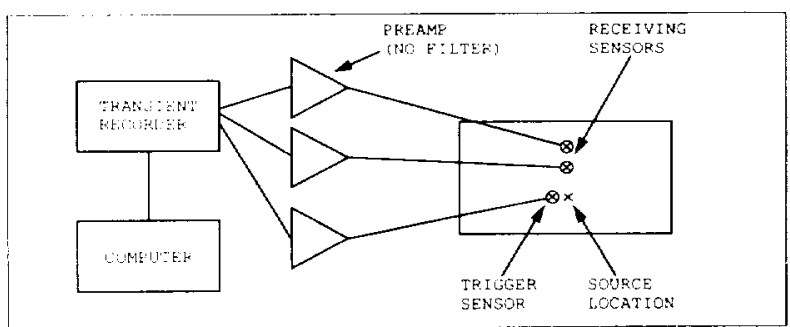

Fl(i. 3. Experimentil setup fur flexural velocity measurements in compenite plittes.

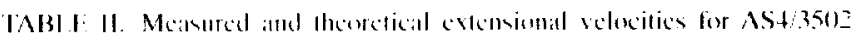

\begin{tabular}{|c|c|c|c|}
\hline 1 aminate & $\begin{array}{l}\text { Dircelion on } \\
\text { propalgitliont }\end{array}$ & $\begin{array}{l}\text { Mcilsured } \\
\text { reloxily |mos) }\end{array}$ & 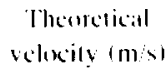 \\
\hline \multirow[t]{3}{*}{$\left|11_{1,}\right|$} & 11 & $4(1 \geq 0)$ & 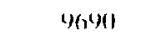 \\
\hline & 45 & 3510 & $71 H 14$ \\
\hline & $(4)$ & $27(101$ & 251111 \\
\hline \multirow[t]{3}{*}{$|11, \cdot 0| \|_{1} \mid$} & (1) & (1.38) & 70157 \\
\hline & 45 & 52111 & $54(24)$ \\
\hline & 41 & 7.31111 & 70187 \\
\hline \multirow[t]{3}{*}{$|11.011|_{4}$} & 11 & 0,5501 & 71187 \\
\hline & 45 & $5(120)$ & 540,4 \\
\hline & 4 & 6450 & $711 \times 7$ \\
\hline \multirow[t]{3}{*}[11.45.45.911]{.} & 0 & 0,15511 & 6.321 \\
\hline & 45 & $5(1)(9) 1$ & 0.321 \\
\hline & 1)(1) & 57511 & $(132)$ \\
\hline
\end{tabular}
graphitcopers lanminates.

center of the plate as possible to minimize reflections.

Prior fo computing the fist Fourier transform (FFT) to determine the phase, the higher-frecuency extensional mode and the reflections arriving later in the flexural mode were zeroed out in the computer. Previous Fourier analysis of the flexural mode signals when digitized at much higher simpling frequencies (10) MHz) showed that the maximum frequency component in the tlexural mode was about $200 \mathrm{kll} \%$ Thus. aliasing was nol a concern evell at the low-sampling frequency of I Mlly.

\section{RESULTS AND DISCUSSION}

The measured and theorelical extensional velocities for all four laminates are shown in Tahle II. The measured velocities were consistently lower than the predicled velocities with the exceptions of the 9(1)-deg measurements in the $\left|0_{16}\right|$ and the $\left.\left[10_{4}, 90\right]_{4}\right]$, plates and the 45-deg measurement in the $\left[11_{10}\right]$ laminate. With these exceptions. the measured velocilies were all in the range from $2 \%$. $10 \%$ less than the theoretical velocities. This would seem to indicate that the mantfacturer's properties used in the theoretical calculations were somewhat higher than those in the actual material. Variations in material properties are common for these materials and are due to fiber volume variations, cure processing variations. and variations in resin chemistry. The 45-deg [0 10$]$ measurement was much less than theoretically predicted. It is not known why this discrepancy occurred and further investigation is needed. The remaining two exceptions, propagation at 90 deg in the $\left[00_{10}\right]$ and the $\left.[0)_{4}, 9()_{4}\right]$, laminates, were only slightly higher than predicted values.

The average measured flexural velocities for the ()-, 45-, and 90 -deg directions in the $\left.[0)_{10}\right]$ graphite epoxy plate are plotted in Figs. 4-6 with the standard deviation of the measured values indicated by crror bars. The predicted velocity dispersion curves for (PT and Mindlin plate theory are also shown in these plots. The agreement hetween measurement and Mindlin plate theory is excellent for the 9()-deg propagation direction. For propagation at 45 and 0 deg. the measured values are consistently less than those predicted by 


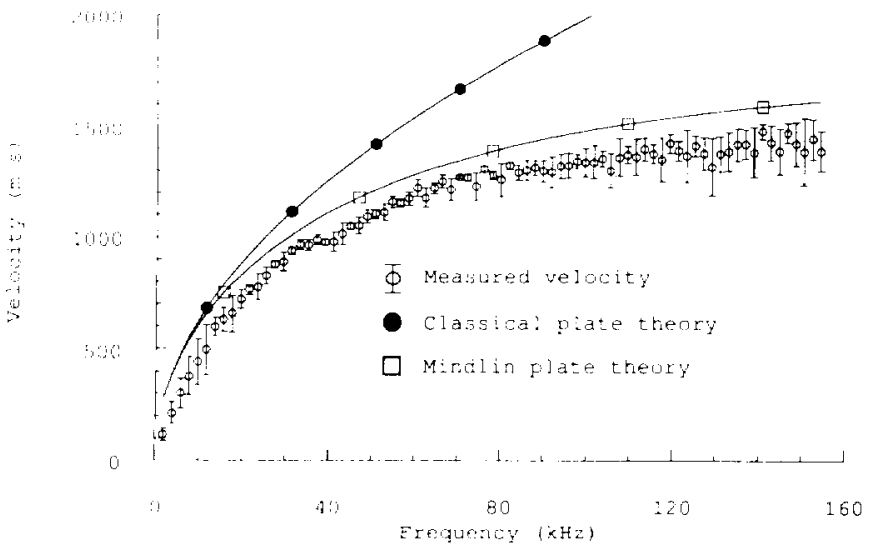

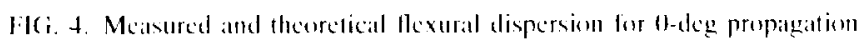
in $\left|0_{\mid t 1}\right|$ graphitcipoxy plate.

theory. The discrepancy at () and 45 deg is consistent with the differences between theory and experiment observed for the extensional mode.

The effect of shear and rotatory inertia is clear when C ${ }^{\prime} T$ and Mindlin plate theory are compared in these plots. They are in agreement at very low frequencies in all cases where the approximations of CPT are valid. The discrepancy increases with increasing frequency as the velocity predicted hy (PT increatses willout bound.

It is also apparent that the difference between Mindlin plate theory and ( $P$ T is much greater for the ()- and 4.5 -deg directions than for the 9h-deg direction. This is expected since the shear modulus is much smatler in comparison to the Young's modulus in those directions. (PT is halsed on the assumption of no shear deformation which implies an intinite shear modulus. Thus, better agreement is provided by ('P' when the ration of the shear modulus to the Young mo molulus is larger.

$A$ plot of the measured velocities and the Mindlin plate theory predictions for the $[0.90] \ldots$ plate is shown in Fig. 7. In order to better view this complicated graph, the ('P'T predictions and the experimental uncertaintics are not shown in the plot. In this plate, the measured velocities were less than theoretical predictions for all three directions of propagation.

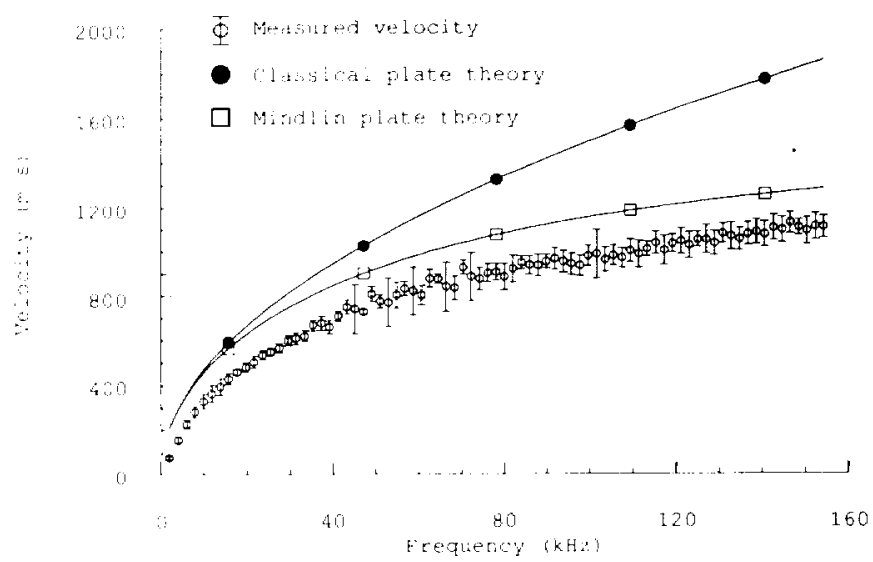

FIc; 5. Measured and thentedical flexural dispersion for 4.5-deg propagation in $\left|0_{16}\right|$ graphituepoxy plate.

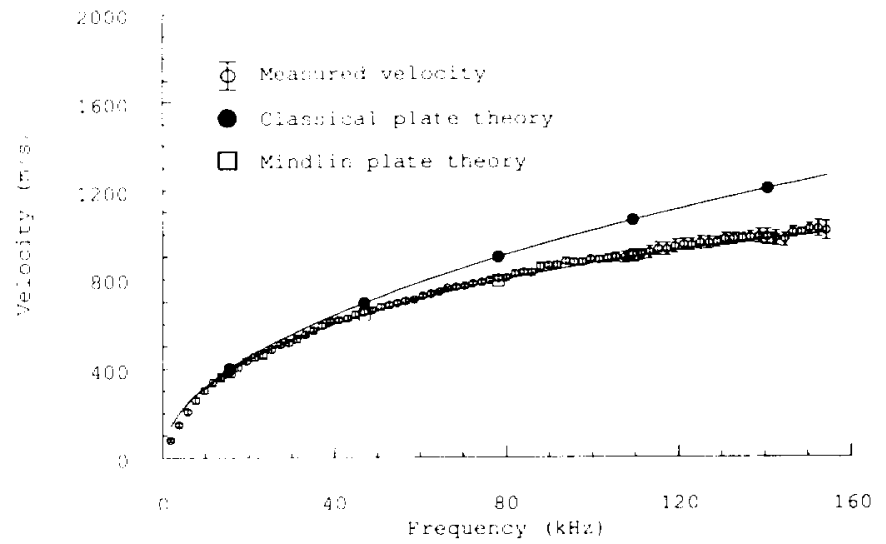

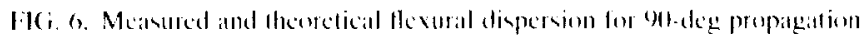
in $\left|t_{k}\right|$ graphitespers plate.

However, the measured and theoretical velecities oceurred in the same order with the (1-deg velocity being the largest. followed nexi by the 9)-deg velecity, and with the 45-deg velocity the smallest. The results for the other two liminates were similar with the measured velocities consistently less that predicted by theory. This again seems to indicate that lhe actual material properties are less than the nominal propertics used in the theoretical calculations and is consistent with the extensional measurements.

In summary, the velocity of the extensional plate mode and the dispersion of the flexural plate mode was meisured along threc directions of propagation in four graphite epoxy laminates. Theorelical predictions of the dispersion of these modes were made with CPT and Mindlin plate theory. CPT yielded good agreement with the extensional velocity but was shown to be of limited value in predicting the dispersion of the flexural mode hecituse it assumes that the effects of shear deformation and rotatory inertia are negligible. Mindlin plate theory. which includes these effects, gave much better agreement with the measured flexural dispersion. However, there was a consistent discrepancy between theory and experiment believed to be due to variations in actual material properties from those used in the calculations.

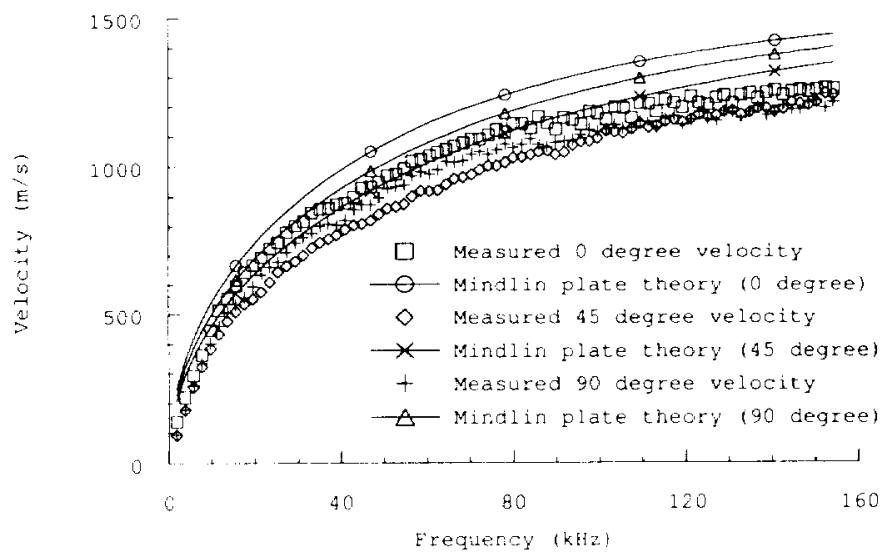

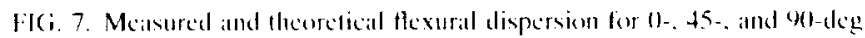
propagatom directions in $[1], 90 \mid$, graphiteceaxy plate.

W. H. Prosser and M. R. Gorman: Plate mode velocities 


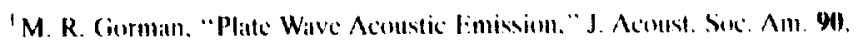
$35 X-364(199(1)$

:M. R. Ciorman and W. H. Prosser, "AE Source Oricatation by Plate Wave Analysis," J. Acoust. Enission 9(4), 2K.3-28K (I)Wl).

${ }^{3}$ M. R. Cormatn and S. M. Ziola, "Plate Waves Produced hy Transverse Matrix Cracking." Ultrasonics 29. 245-2.51 (149)1.

${ }^{+}$W. D. Smith, Jr., "Acouslic Emission From Spectrum Fatigute Cracks in 7075 Aluminum." Masters thesis. Naval Pustgratuatte Schowl (190)(1).

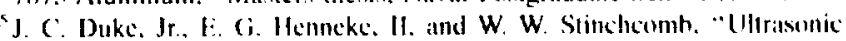
Stress Wave Characterization of cimposite Materials." NASA Comtractur Report 3476 (1986).

"N. A. Schumacher. P. H. Cilen. and ( C. P. Burger. "Analysis of Transicul lamh Waves on Mctal Plates, Compensite Pands and curved Menthers." Rev. Progress Quimt. NDI: 11B. 1564-1576 (140)|1.

'S. M. Zionla and M. R. Ciornuan, "Source Ionation in Thin Plates (Ising Cross-Correlation," J. Acoust. Sik: Am. 90, 2551-2556 (1491).

"A. K. Mal. M. R. Gorman, and W. H. Prosser. "Material Chatracterizalion or Composite laminates Using low-Frequency Plate Wave Dispersion Data. Rev. Progercss Ouant. ND)l 11B. 1451-14.58 (100)1).

"M. Veidt and M. Sayir. "IExperimental Evaluation of cilohal composite Laminate Stiffinesses by Strustural Wive Propagitlon," J. ( impos. Malcr. 24. $6 \times 8-7016,119011$

"G. D. Dean. "The Use uf Plate Bending Waves for liastic Properly Delermination of Polyners." Nitlonal Physicall laborallory Report I)MA $(A)$ I06 (Teddington. Midellasex. UK, 1488).

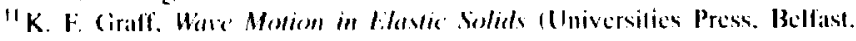
1475).

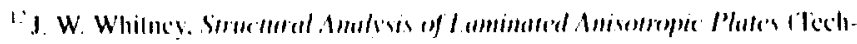
nomic, lancasler. Pentusvlvania. 19x71.

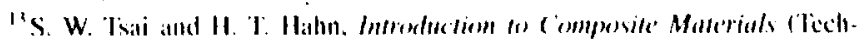
numic, Lancaster, Pennsylvania, IUxil).

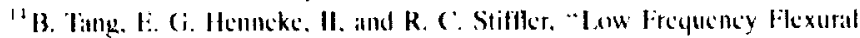
Wave Propagation in laminated composite Plates," Proceedings an a Wurkshop on Acousta-Ultrasonics: Theory and Applications, B3lacksharg Virginiat. pp. $4.5-0.511987)$

I'P. ('. Yang. ('. H. Norris, and Y. Stavsky, "Hastic Wave P'ropalgatlon in Helerogencous Plates". Inı. J. Solids Struct. 2, $60,5-6 \times 4$ (1966)

" R. D). Mindlin. "Intluence of Rotitury Incrtia and Slucar on llexaral Molions of Isotropic. Elastic Plates," J. Appl. Mech. 2. 31 - $38 / 1951)$.

"W. H. Prosser. "The Propagation Charatcheristice of the Plate Moxtes of Acoustic Fmission Waves in Thin Aluminum Plates and Thin Ciraphite

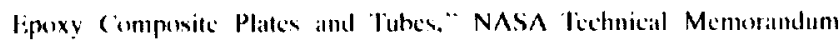
104187119411.

${ }^{1 \times}$ W. Saches and Y. II. Pan, "()n the Delermination of Phase and (iroup Velesilies of Dispersive Waves in Solids," J. Appl. I'hys. 49. 4.3201.4327 (1978).

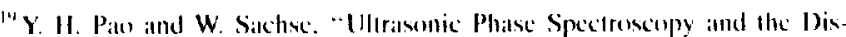
persion of talastic Waves in Solids," Procecdings of the Nindh Internalional (ingress an Acoustics. Madrid. Spain, 7.3411977$).$

"1). Alleync and P. (atwley. "A Two-bimensional Fourier Tinnstorm

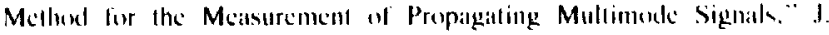
Acount Six. Am. 89. $1159-116 \times 110411$ 

